\title{
Nanodispositivos para la prevención y tratamiento de enfermedades cardiovasculares
}

\author{
Juan Manuel Vélez-Reséndiz y Juan Jesús Vélez-Arvízu² \\ ${ }^{1}$ Instituto Politécnico Nacional, Sección de Estudios de Posgrado e Investigación, Escuela Superior de Medicina, Laboratorio Multidisciplinario de \\ Nanomedicina; ' Universidad Nacional Autónoma de México, Facultad de Ciencias. Ciudad de México, México
}

\begin{abstract}
Resumen
Actualmente las enfermedades cardiovasculares representan la principal causa de morbimortalidad en el mundo; la aplicación de la nanotecnología es una gran promesa en su prevención y tratamiento. Se están desarrollando nanodispositivos para la liberación dirigida y controlada de medicamentos en sitios específicos en el organismo, por ejemplo, en células, tejidos, vasos sanguíneos y el corazón, así como para el diagnóstico, detección temprana de enfermedades cardiovasculares y tratamiento individualizado de pacientes. Otra posible aplicación de los nanodispositivos es la liberación de fármacos para corregir el mal acoplamiento de proteínas defectuosas. Con potentes superefectos, las nanopartículas deberán ser capaces de provocar efectos terapéuticos a bajas dosis en periodos prolongados. La fabricación de nanodispositivos y nanoacarreadores deberá llevarse a cabo con un enfoque integral que tome en cuenta las propiedades generales, con la finalidad de evaluar la biocompatibilidad y, en consecuencia, evitar efectos adversos y tóxicos. La investigación intensificada en este campo ayudará a reducir significativamente la morbimortalidad provocada por las enfermedades cardiovasculares.
\end{abstract}

PALABRAS CLAVE: Nanomedicina. Nanoacarreadores. Nanodispositivos. Nanotecnología. Sistema de liberación de fármacos. Enfermedades cardiovasculares. Fármacos inteligentes.

\begin{abstract}
Currently, cardiovascular disease represents the main cause of morbidity and mortality worldwide; the application of nanotechnology holds great promise for its prevention and treatment. Nanodevices ("smart drugs") are currently being developed for directed and controlled delivery of drugs to specific sites in the body, such as cells, tissues, blood vessels and the heart, as well as for diagnosis and early detection of cardiovascular conditions and patient-individualized treatment. Another application of nanodevices is the delivery of drugs to correct defective protein bad coupling or binding. With potent super-effects, nanoparticles should be able to elicit therapeutic effects at lower doses and prolonged periods. The manufacture of nanodevices and nanocarriers should be with a comprehensive approach that takes general properties into account in order to assess for biocompatibility and, therefore, avoid adverse and toxic effects. Intensified research in this field will help to significantly reduce morbidity and mortality caused by cardiovascular disease.
\end{abstract}

KEY WORDS: Nanomedicine. Nanocarriers. Nanodevices. Nanotechnology. Drug delivery systems. Cardiovascular diseases. Smart drugs.

\section{Introducción}

Las enfermedades cardiovasculares (ECV) afectan el corazón y los vasos sanguíneos del cuerpo humano; de acuerdo con la Organización Mundial de la Salud constituyen la principal causa de morbimortalidad en países desarrollados y en desarrollo. Cada año mueren más personas por ECV que por cualquier otra
Correspondencia: Juan Manuel Vélez-Reséndiz E-mail: jvelezr@ipn.mx
Fecha de recepción: 13-03-2016

Fecha de aceptación: 01-11-2016

DOI://dx.doi.org/10.24875/GMM.18002507
Gac Med Mex. 2018;154:358-367

Disponible en PubMed www.gacetamedicademexico.com 
enfermedad: 23 millones de personas murieron por ECV en 2011, 30 \% de la totalidad de muertes registradas en el mundo.' Se pronostica que las ECV continuarán siendo la principal causa de muerte para 2030,1,2 un negro escenario que debe ser enfrentado mediante las ventajas y avances en la nanotecnología.

La nanotecnología se define como la síntesis de partículas y la fabricación de dispositivos a una escala que les permite actuar a nivel molecular. Debido a sus propiedades derivadas del tamaño, las nanopartículas ofrecen la posibilidad de desarrollar nuevas herramientas para el diagnóstico y tratamiento de las ECV. La nanomedicina involucra el uso de moléculas a escala de 10 a $100 \mathrm{~nm}$ para propósitos de diagnóstico, tratamiento y prevención. ${ }^{3}$ Entre las potenciales aplicaciones clínicas se encuentra la liberación dirigida y controlada de fármacos para maximizar la actividad terapéutica y minimizar los efectos adversos. La mayoría de las investigaciones están enfocadas al diagnóstico y tratamiento de las ECV utilizando nanopartículas (NP), por ser la causa más frecuente de morbimortalidad. Sin embargo, los investigadores están estudiando las NP para uso en síndromes coronarios agudos, procedimientos de revascularización, aneurisma aórtico, insuficiencia cardiaca, eventos cerebrovasculares, hipertensión, trombosis y otros padecimientos cardiovasculares. El diseño y caracterización de nanosistemas, validaciones preclínicas y clínicas y análisis toxicológicos son ejemplos de estudios en desarrollo, cuya finalidad es evitar la aparición de placas ateromatosas y eventos cerebrovasculares mediante imagenología y tratamiento preventivo. ${ }^{4}$

Debido a su tamaño y propiedades fisicoquímicas de adaptación, los nanomateriales son estructuras con aplicación biomédica en imagenología, diagnóstico, tratamiento y medicina regenerativa. ${ }^{5}$

El tratamiento actual de las ECV incluye fármacos, la intervención coronaria percutánea con endoprótesis y la cirugía de revascularización coronaria. La mayoría de las complicaciones se derivan de estas modalidades terapéuticas, como la toxicidad sistémica por el medicamento o la trombosis con intervención coronaria percutánea. La nanomedicina ha surgido como una estrategia para evitarlas. ${ }^{6}$

El presente trabajo describe algunos de los avances más recientes de la nanomedicina en las ECV. A corto plazo, existe la posibilidad de desarrollar nanoacarreadores tanto para la administración, liberación dirigida y controlada de fármacos como de procedimientos diagnósticos destinados a prevenir, atenuar y curar. A largo plazo, robots para nanocirugía y el uso de NP para impedir o corregir defectos y el mal acoplamiento de proteínas. La investigación en estos campos podría disminuir la morbimortalidad de las ECV.

Además de la capacidad para actuar a bajas dosis y aumentar la ventana terapéutica, las NP pueden provocar respuestas al estrés celular que compensen los mecanismos sistémicos por más tiempo. ${ }^{7}$ Los nanodispositivos podrían ingresar al tejido cardiaco y a la circulación sanguínea para liberar directamente el principio activo en sitios específicos, lo cual reduciría aún más la toxicidad e incrementaría los efectos terapéuticos. Finalmente, la unión y el mal acoplamiento de las proteínas en nanoagregados que varían en morfología y tamaño representan un área relativamente nueva de investigación. Varios metabolitos intracelulares que controlan el acoplamiento de las proteínas, así como las interacciones con otras proteínas pueden ser objetivos para las NP., El entendimiento fundamental de los procesos moleculares que conducen al mal acoplamiento y a la autoagregación de las proteínas implicadas en la aparición de las ECV podría proporcionar más información acerca del funcionamiento de estas, lo que ayudaría al diseño de nuevas estrategias terapéuticas.

\section{Aneurisma aórtico}

La identificación del paciente con aneurisma aórtico abdominal es útil para el tratamiento. El grosor, la forma de la pared arterial y el trombo intraluminal son de gran importancia para la predicción de la ruptura de los aneurismas aórticos abdominales. La definición de los contornos interno y externo de la pared de la aorta permite distinguir entre la pared del vaso y el trombo intraluminal. Este método funciona en un modelo de forma activa e información estadística de la textura. ${ }^{9}$ Existe alta correspondencia entre las medidas automáticas y manuales de las áreas de la pared del vaso. Los nanodispositivos facilitan este procedimiento.

\section{Infarto agudo del miocardio e insuficiencia cardiaca}

La insuficiencia cardiaca es un síndrome complejo derivado de múltiples causas heredadas 0 adquiridas de la estructura o función cardiaca. EI IAM es causa de la insuficiencia cardiaca y continúa siendo un problema creciente en términos de morbilidad, mortalidad y costos. Los tratamientos farmacológicos han demostrado ser eficaces en la disminución y regresión de la hipertrofia cardiaca, remodelación miocárdica y 


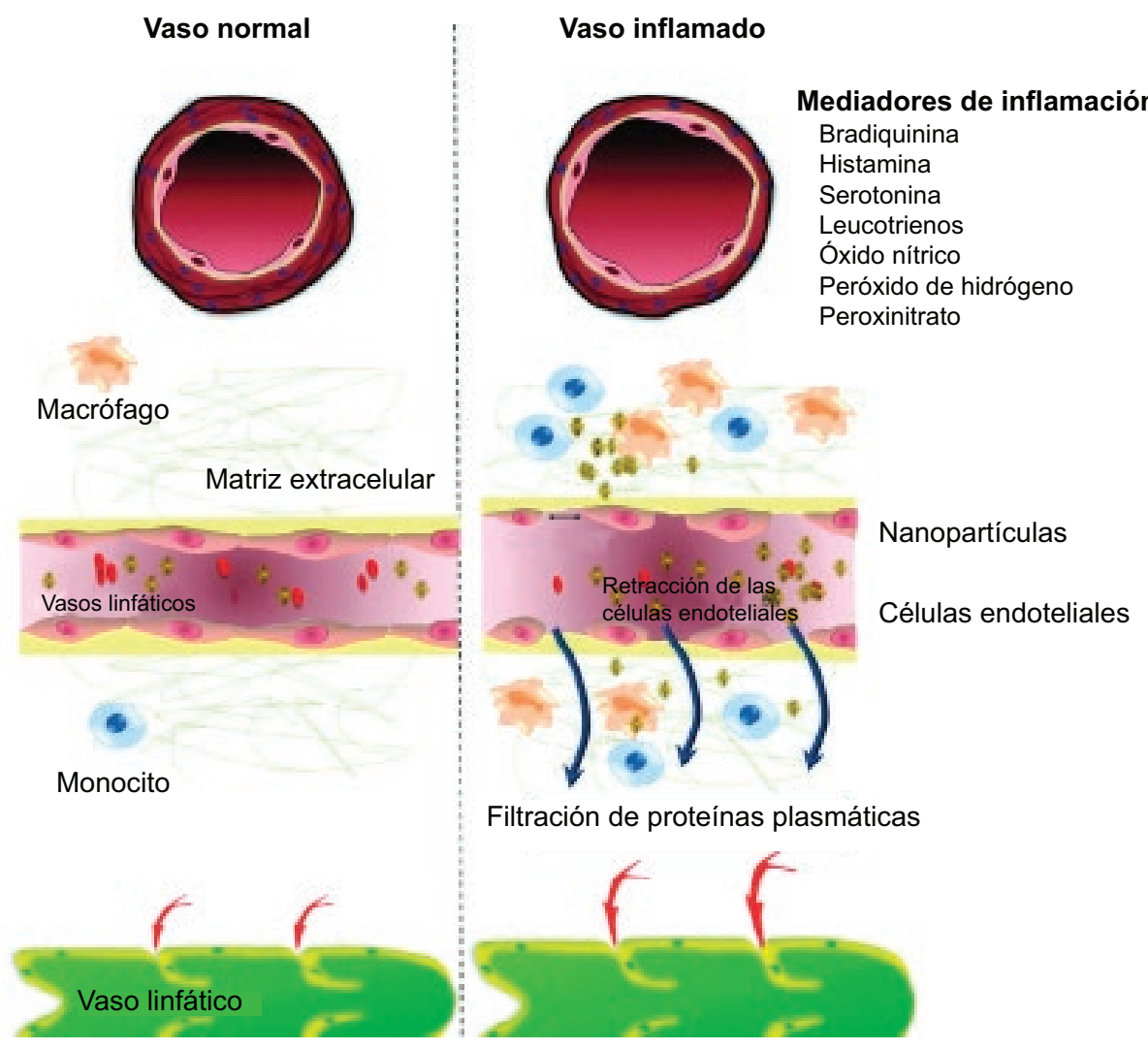

Figura 1. Comparación entre un vaso normal y un vaso inflamado que fue posible con el uso de nanopartículas.

disminución de la morbimortalidad; ${ }^{10} \sin$ embargo, su pronóstico es pobre.

Las NP magnéticas pueden ser multifuncionales para una liberación prolongada y selectiva de los fármacos. Las NP polifuncionales con PLGA (poly lactic-co-glycolic acid) han sido diseñadas con una estructura nuclear acorazada que actúa como un vector robusto para la encapsulación y liberación de péptidos y proteínas. Además, el factor de crecimiento endotelial vascular podría ser liberado de forma específica en el tejido cardiaco del miocardio infartado, para ayudar a regenerar la vasculatura y las células madre mesenquimales en IAM. ${ }^{11}$

En los pacientes con dolor torácico estable inducido por ejercicio, la angina microvascular es el diagnóstico más probable. Hallazgos clínicos y resultados de las pruebas de diagnóstico sugieren que la angina de pecho es causada por disfunción coronaria microvascular, pero se requiere el diagnóstico definitivo para la demostración del deterioro por la disfunción coronaria microvascular o vasoconstricción aumentada. ${ }^{12}$ El uso de las nanopartículas es de gran utilidad en el análisis, diagnóstico y tratamiento.

\section{Inflamación aguda y crónica}

Los nanoacarreadores pueden ser usados para el tratamiento de la inflamación en los revestimientos de las células endoteliales de los vasos sanguíneos. Los nanoacarreadores pueden ser utilizados para ser dirigidos a células endoteliales y, por tanto, facilitar la penetración a este tejido. Varios marcadores de superficie endotelial, incluyendo peptidasas y moléculas de adhesión, se han identificado como objetivos blancos para este propósito. ${ }^{13}$ La unión de los nanoacarreadores a estas moléculas permite la liberación selectiva de fármacos a través y dentro de la capa endotelial, proporcionando efectos terapéuticos inalcanzables por sus contrapartes no dirigidos (Figura 1).

Hay receptores específicos que pueden atenuar la inflamación mediante la modulación en la expresión de genes inflamatorios clave. Estos receptores y sus ligandos son objetivos atractivos para la atenuación de la inflamación del tejido endotelial vascular mediante NP poliméricas. ${ }^{14,15}$ Para el monitoreo y tratamiento de ECV inflamatorias pueden diseñarse estrategias de liberación dirigida y controlada de 
fármacos o agentes de contraste a sitios específicos con NP basadas en polímeros biodegradables.

Además, algunos agentes antiinflamatorios (por ejemplo, bioterapéuticos) requieren la liberación precisa dentro de los compartimientos subcelulares. La liberación óptima en los sitios deseados podría mejorar el efecto de los agentes antiinflamatorios; con este propósito han sido diseñados y explorados diversos nanoacarreadores para su liberación en células endoteliales. ${ }^{16}$ Es necesario un análisis riguroso para cuantificar los efectos terapéuticos-adversos y costo-beneficio y la utilidad clínica en el manejo y control de la inflamación aguda y crónica.

Actualmente se ha reportado que la superóxido dismutasa conjugada con anticuerpos provee la liberación dirigida dentro de los endosomas endoteliales, mitigando la activación inflamatoria endotelial por citocinas y agonistas de receptores tipo Toll. ${ }^{17}$ Hay evidencia de que el papel principal de la superóxido intracelular es mediar la activación proinflamatoria del endotelio. Los nanoacarreadores ayudarían a elucidarlo.

\section{Aterosclerosis}

La aterosclerosis es una enfermedad sistémica inflamatoria, crónica y progresiva que se inicia por la disfunción endotelial y posteriormente se amplifica por el estrés oxidativo, el depósito de lípidos y el reclutamiento de monocitos. Esta interacción ocurre entre diferentes células, factores quimiotácticos, moléculas de adhesión y lipoproteínas de baja densidad en el subendotelio. Los sistemas nanoparticulados con aplicaciones diagnósticas y terapéuticas para la liberación dirigida y controlada en sitios específicos de lesiones ateroscleróticas, así como nanomateriales adecuados para implantes cardiovasculares pueden ofrecer soluciones a deficiencias terapéuticas actuales. Diversos acontecimientos han mostrado el potencial de la nanomedicina para la prevención, identificación y tratamiento de la aterosclerosis..$^{18,19}$ que van desde estrategias "nanoteranósticas" para placas vulnerables, hasta nanoporos y nanopartículas basadas en la liberación de fármacos en la endoprótesis, con el propósito de lograr un equilibrio entre la eficacia y la toxicidad potencial de los sistemas fabricados con nanotecnología. ${ }^{20,21}$

Una alternativa es el desarrollo de una apoptosis dirigida con una NP que imite a la lipoproteína de alta densidad y transporte agentes de contraste para la detección temprana de placas vulnerables, lo que permitiría a los pacientes recibir tratamiento preventivo que potenciaría los efectos protectores vasculares de la lipoproteína de alta densidad. ${ }^{22}$ Estudios de imagenología molecular cardiovascular han sido aplicados en estudios in vivo empleando nanoacarreadores que son liberados y dirigidos hacia los componentes del sistema inmunitario que participan en el desarrollo de la placa aterosclerótica. ${ }^{23}$

Las micelas poliméricas son estructuras autoensambladas de polímeros anfifílicos que tienen un núcleo hidrófobo y una coraza hidrofílica, constituyen una clase de vehículos de liberación que ha sido investigados para varias aplicaciones biomédicas. Polímeros anfifílicos basados en moléculas de azúcar (PABA) han sido empleados para la liberación de fármacos anticancerígenos vía encapsulación física dentro de micelas PABA y una conjugación química para formar profármacos PABA capaces de formar micelas. Los PABA son excelentes sistemas de liberación para la estabilización liposomal y tienen una única bioactividad, que podría ser útil para el tratamiento y prevención de la aterosclerosis. ${ }^{24-26}$

\section{Ateroma carotídeo}

Actualmente se utilizan nanodispositivos para el estudio de pacientes con ateroma carotídeo. ${ }^{4,27}$

\section{Hipertensión}

Se han desarrollo materiales biológicos combinados para aplicaciones médicas específicas. Una de las áreas de investigación es la detección específica de secuencias de ADN para el diagnóstico temprano de enfermedades genéticas utilizando NP que contienen nanosensores génicos. Los nanodispositivos sensores génicos típicos emplean electrodos de biorreconocimiento que contienen sondas inmovilizadas para capturar ADN, capaces de hibridarse con secuencias específicas dirigidas de ADN. Genosensores eficientes tipo sándwich basados en complejos de AND-NP de oro pueden ser aplicados eficientemente para la detección de polimorfismos génicos de la presión arterial sistémica. ${ }^{28}$

\section{Imagenología en tercera dimensión}

Una nueva área denominada "liberación de fármacos guiada por imagenología en 3D" (LFGI), que actualmente combina el potencial de las ciencias radiológicas con la liberación dirigida de fármacos para el tratamiento, promete cumplir los retos de la medicina personalizada. 
Actualmente, la LFGI es utilizada para liberar fármacos alrededor de los tumores y de esta forma incrementar su efecto, así como para cuantificar distintos biomarcadores que podrían poner en riesgo a los pacientes. El objetivo principal de la LFGI es utilizar la imagenología para maximizar el tratamiento efectivo de tejidos dañados y minimizar la exposición a fármacos con la finalidad de reducir su toxicidad. ${ }^{29}$

Modalidades de imagenología en 3D aprovechan hasta la última pulgada del espectro de energía. En estas modalidades se incluye la imagen por resonancia magnética, la tomografía computarizada por rayos $\mathrm{X}$, el ultrasonido y métodos basados en la luz (endoscopia y tomografía de coherencia óptica). Las modalidades para investigación incluyen varias técnicas de microscopia de luz (confocal, multifotón, reflexión interna total, microscopia por fluorescencia de super-resolución), microscopia electrónica, imagenología por espectroscopia de masas, tomografía por fluorescencia, bioluminiscencia, quimioluminiscencia, variaciones de la tomografía de coherencia óptica e imagenología optoacústica, entre otras. ${ }^{30}$

Actualmente, las NP para imagenología molecular son empleadas en endoprótesis liberadoras de fármacos y en técnicas para la detección temprana in vivo y ex vivo. ${ }^{31}$ Los miocitos contraídos pueden ser analizados por imagenología en 3D con microscopia óptica de alta resolución espacial y temporal con el corazón latiendo, lo que permite la visualización de los sarcómeros de manera independiente y medir el ciclo contráctil de un cardiomiocito en forma individual, lo cual ha sido posible por la eficiente estabilización del tejido, una aproximación prospectiva de la sincronización cardiaca en tiempo real, un algoritmo para capturar imágenes en movimiento libre de artefactos durante el ciclo cardiaco completo y un protocolo fluorescente de tinción fluorescente de la membrana. ${ }^{32}$ La cuantificación de la función contráctil del cardiomiocito in vivo permite diversas posibilidades para la investigación de las enfermedades miocárdicas y para la intervención terapéutica a nivel celular.

La microscopia de imagen intravital (por ejemplo, imagen de resolución microscópica en animales vivos) se ha convertido en una herramienta indispensable para estudiar la microdinámica celular en las ECV. La alta resolución espacial y temporal, combinada con una penetración profunda y la capacidad de visualización multirreportada hace de la microscopia intravital fluorescente muy conveniente para la imagenología en 3D cardiaca, sin embargo, el movimiento del tejido causado por la contracción cardiaca y la respiración limitan considerablemente su uso. Como resultado, las preparaciones celulares in vitro o modelos de cultivos celulares de corazón no contráctiles son más utilizados. Los métodos se basan en la estabilización mecánica, en algoritmos de procesamiento de imágenes, en esquemas de adquisición obtenidos/disparados 0 una combinación de ambos. ${ }^{33}$ Su uso permitirá incrementar el entendimiento de la biología cardiaca, elucidando la función del cardiomiocito y sus interacciones en el interior del organismo in vivo y mejorar la prevención y tratamiento de las ECV.

\section{Métodos y técnicas}

Actualmente, la imagenología por tomografía computarizada permite evaluar las lesiones coronarias, pero carece de la capacidad para medir la extensión transmural de la cicatriz coronaria. ${ }^{34}$ Otro método es la conjugación de péptidos poliméricos y la encapsulación física, que se divide en técnicas basadas en surfactantes y técnicas basadas en acarreadores poliméricos. Las primeras predominantemente emplean liposomas, microemulsiones y NP lípido-sólido. ${ }^{25,27}$ Los sistemas nanodirigidos, como los nanoacarreadores recubiertos con polímeros, albúmina o partículas lípido-sólido, han sido empleados como transportadores in vivo en modelos animales; los fármacos atrapados dentro de los acarreadores son liberados selectivamente en su objetivo o depositados en la superficie de capas lipídicas de células cardiacas y en vasos sanguíneos (Figura 2).

\section{Nanoacarreadores para la liberación de fármacos}

Existen dos grandes retos para la nanoliberación sistémica efectiva: la penetración limitada a través del endotelio vascular y la captación por el sistema retículo endotelial, que impiden la eficacia de la liberación de las NP dentro de los tejidos. Si bien el diseño de las NP con vida media prolongada en la circulación es esencial, no es suficiente para que las NP crucen el endotelio vascular. Los actuales sistemas de nanoliberación se fundamentan en el intercambio pasivo transvascular y la acumulación tisular. Se requieren grandes dosis para crear grandes gradientes de concentración que conduzcan pasivamente a las NP a través de la interfase tejido-circulación sanguínea. Hasta el momento, la acumulación pasiva ha resultado en una dosis fraccional de NP que penetra el tejido blanco, lo que inevitablemente 

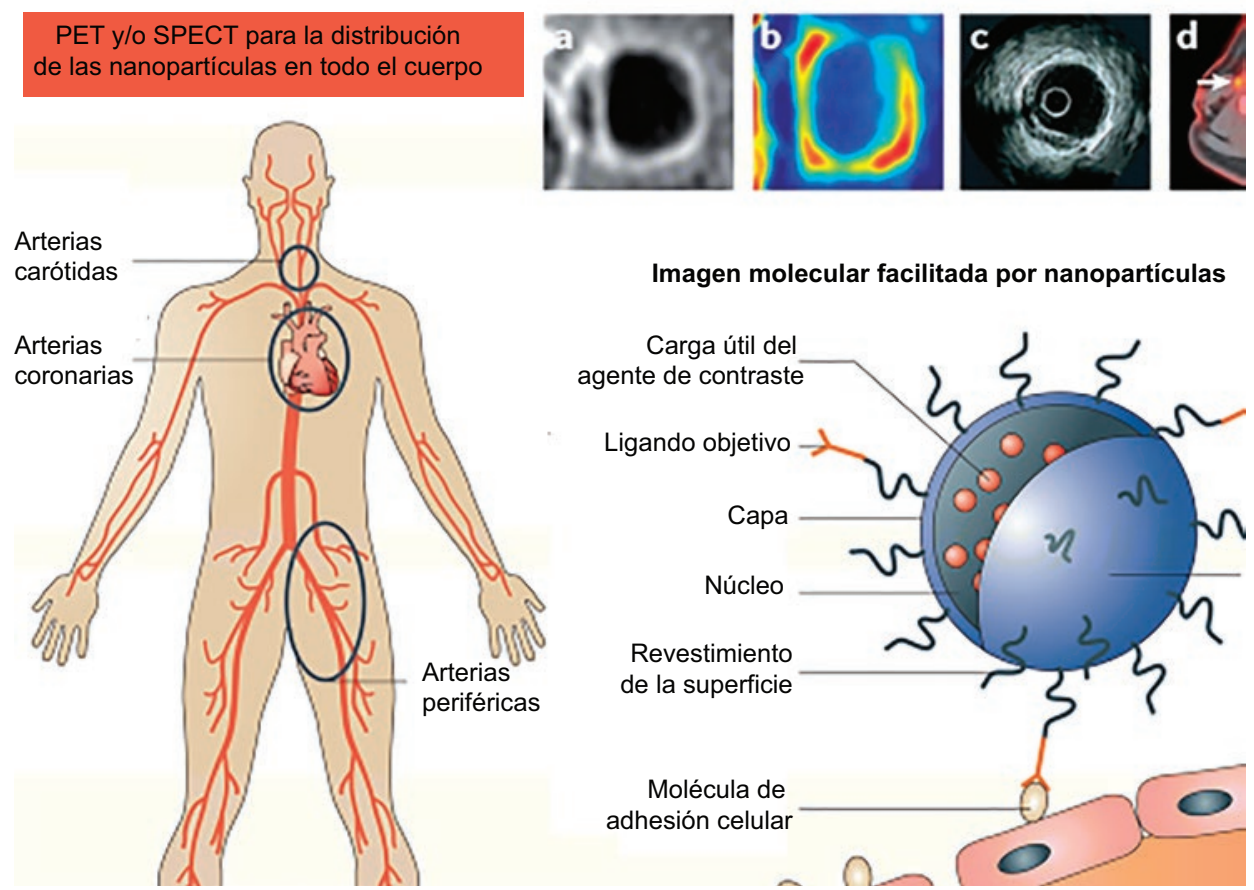

Imagen molecular facilitada por nanopartículas

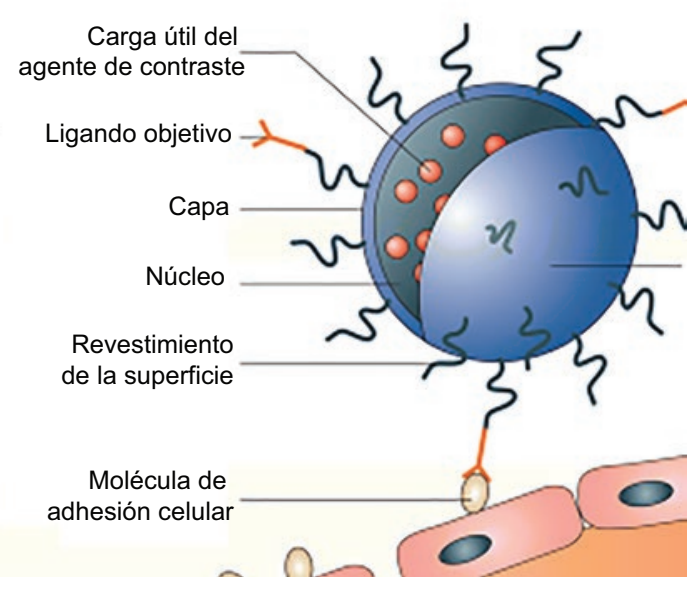

Figura 2. Técnicas para el tratamiento de ECV mediante nanopartículas.

disminuye la eficacia terapéutica y agrava los efectos adversos potenciales. La liberación activa de las NP dirigidas a través del endotelio vascular podría incrementar significativamente el índice terapéutico y disminuir los efectos adversos de los sistemas de liberación de fármacos basados en NP. ${ }^{35}$ Las rutas de transporte transendotelial, tales como la caveola, podrían proporcionar una solución efectiva tanto para el destino específico como para la liberación efectiva de las NP (Figura 3).

El uso de sistemas de liberación de medicamentos nanoparticulados (NDDS, por sus siglas en inglés) para incrementar la efectividad de fármacos in vivo está bien establecido, el desarrollo de NDDS multifuncionales y sensibles al estímulo es un área activa de investigación. Estos NDDS pueden tener tiempos de circulación prolongados, dirigirse a sitios específicos de la enfermedad, incrementar la liberación intracelular del fármaco y responder a estímulos locales, por ejemplo, la liberación de un fármaco atrapado o el derramamiento de una capa protectora, lo que facilitaría la interacción entre los nanoacarreadores de los fármacos cargados y las células o tejidos blanco. ${ }^{36}$ Además, los restos de formación y el contraste de las imágenes pueden ser conectados a estos acarreadores para seguir su biodistribución en tiempo real y su acumulación en células o tejidos blanco.
Por otra parte, los coloides de oro han fascinado a los científicos por más de un siglo y ahora son empleados en química, biología, ingeniería y medicina. En la actualidad, estos materiales pueden ser sintetizados de manera fácilmente reproducible a partir de grupos químicos funcionales aparentemente ilimitados y, en ciertos casos, caracterizados con precisión a nivel atómico. Existen varios ejemplos de pruebas altamente sensibles y selectivas basadas en nanoconjugados de oro. En los últimos años se exploran las posibilidades terapéuticas de tales materiales. Se han desarrollado estructuras que se comportan como agentes de regulación génica, acarreadores de fármacos, agentes para imagenología y agentes terapéuticos fotosensibles, estudiados a nivel celular y en enfermedades comunes como las ECV. ${ }^{37}$ Las propiedades físicas y químicas de estas estructuras les confieren ventajas sustanciales con aplicaciones a nivel celular y molecular.

Existen múltiples barreras a través del organismo que limitan la migración de sustancias xenobióticas a los tejidos, de ahí que una meta en la administración y liberación de fármacos es crear estrategias de transporte que imiten a las de los virus y bacterias. ${ }^{38}$ El empaquetamiento de los fármacos dentro de sus vehículos a escala micro y nano debería permitir cambiar la biodistribución y los tiempos de permanencia 


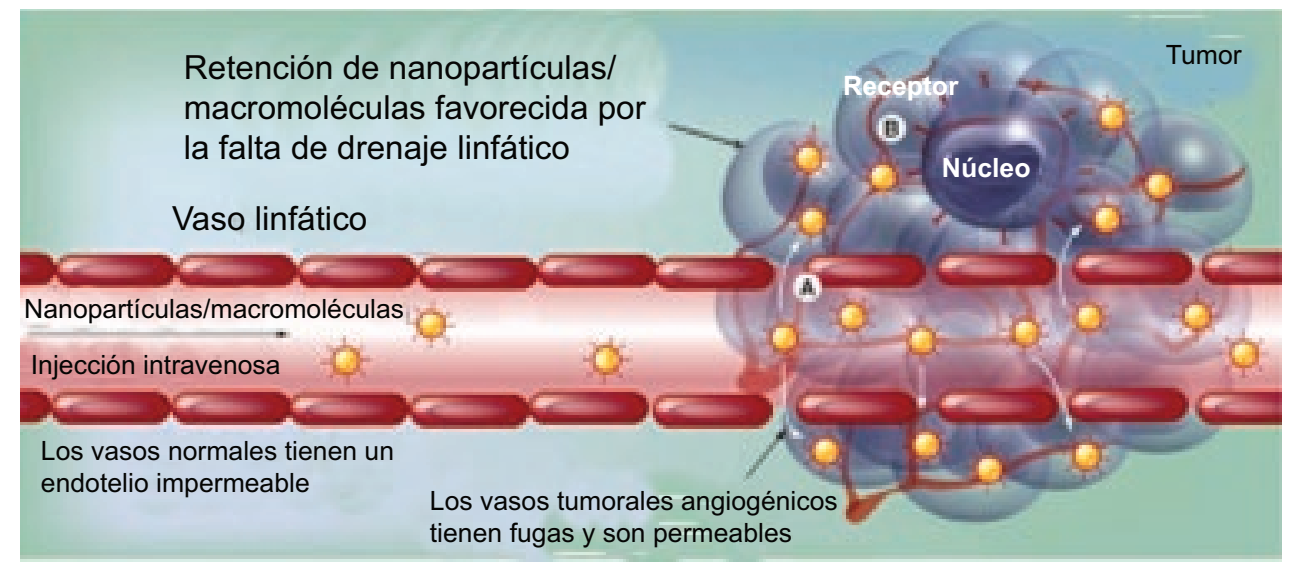

Figura 3. Uso de nanopartículas para la liberación dirigida de fármacos.

de los compuestos farmacológicamente activos. Por ello, el desarrollo de los nanodispositivos para la administración de fármacos involucra típicamente la caracterización de la liberación in vitro, asumiendo que el rendimiento se aproximará a las circunstancias in vivo. Sin embargo, los dispositivos son cada vez más complejos y en los modelos de liberación secuencial de un fármaco es importante confirmar que las propiedades in vivo se correlacionan de manera esperada "a las programadas" y obtenidas in vitro. ${ }^{39}$

Las enzimas antioxidantes (EAO) para el tratamiento de enfermedades relacionadas con el estrés oxidativo son prometedores. Diversos sistemas de nanoacarreadores son desarrollados para abordar y corregirlos dos grandes retos de su aplicación clínica: su pobre localización y estabilidad. En la mayoría de los casos ha existido una compensación entre la masa liberada de la enzima cargada y la capacidad de los acarreadores para proteger a la enzima de la degradación catalítica. ${ }^{40}$ Un método potencial para superar esta limitación es el uso de nanomateriales se silicio mesoporoso ordenados como potenciales nanoacarreadores de las EAO.

Aun cuando se sabe que las EAO, la catalasa y la superóxido dismutasa desintoxican a las especies dañinas de oxígeno reactivas, la utilidad terapéutica de las EAO es obstaculizada por su liberación inadecuada. La modificación de las EAO mediante polietilenglicol y la encapsulación de liposomas recubiertos con este incrementa la biodisponibilidad de la EAO y aumenta sus efectos protectores en modelos animales. Micelas basadas en formulación plurónica formadas con EAO muestran efectos más protectores. Nanoacarreadores basados en copolímeros de PEG protegen a las EAO encapsuladas de la proteólisis y aumentan la liberación en células blanco, tales como el endotelio que reviste la luz vascular. Anticuerpos como determinantes endoteliales conjugados para EAO o acarreadores de EAO proporcionan dirección y liberación intracelular. Los liposomas dirigidos, las proteínas conjugadas y las NP magnéticas liberan EAO en los sitios con presencia de estrés oxidativo en el sistema cardiovascular. Los avances en los nanodispositivos para la liberación de EAO proporcionarán las bases para la comprensión con un enfoque en el campo clínico. ${ }^{27,41}$

\section{Paro cardiaco y eventos cerebrovasculares}

La aplicación de la nanotecnología en la ingeniería de tejidos es una gran promesa. Las NP son empleadas para monitorear a las células injertadas o para incrementar la visualización de los tejidos mediante una modalidad de imagen no invasiva por resonancia magnética, tomografía axial computarizada y escaneo por tomografía de emisión de positrones. Pueden usarse diversos agentes para formación de imágenes por contraste, desde óxido de hierro, compuestos perfluorocarbonados, óxido de cerio o NP de platino hasta puntos cuánticos. Los nanotubos de carbono pueden actuar como andamiajes para el tratamiento del paro cardiaco y los eventos cerebrovasculares mediante células madre. ${ }^{42}$

\section{Trombosis}

Una estrategia para la administración y liberación biomimética de fármacos por parte de los nanoacarreadores podría ser útil en vasos sanguíneos obstruidos por trombosis o embolismo. Una estrategia biofísica podría consistir en dirigir los nanoacarreadores durante la fuerza de roce causada por el estrechamiento vascular (de la misma manera que lo hacen las plaquetas), 
lo que disminuiría las dosis de los fármacos, minimizaría sus efectos adversos y potenciaría su eficacia terapéutica. ${ }^{43}$ Además, los nanoacarreadores in vivo e in vitro han demostrado reducir significativamente la dosis de los fármacos trombolíticos usados. ${ }^{44}$

En la farmacoterapia antitrombótica continúan existiendo dificultades en el manejo de pacientes con alto riesgo de trombosis y hemorragia. La utilidad de los agentes antitrombóticos en estos escenarios está limitada por la inadecuada farmacocinética y el estrecho índice terapéutico. El uso de avanzados sistemas de liberación de fármacos (ADDS, por sus siglas en inglés) podría ayudar a corregir estos problemas. Varios nanoacarreadores, ligandos con afinidad y polímeros recubiertos proporcionan ADDS, con el potencial de optimizar la farmacocinética de los agentes antitrombóticos, la liberación dirigida de fármacos a sitios trombóticos y detectar los cambios patológicos en el microambiente vascular, tales como fuerzas hemodinámicas alteradas, expresión de marcadores inflamatorios y diferencias estructurales entre coágulos maduros homeostáticos y coágulos patológicos en crecimiento.

La liberación de agentes antitrombóticos mediante acarreadores sintéticos biomiméticos, células sanguíneas huésped y proteínas de fusión recombinante activadas en sitios donde se desarrollan los trombos ha resultado alentadora en modelos preclínicos. Por otra parte, el desarrollo y traducción de los ADDS para separar los coágulos de fibrina homeostática mantiene la promesa de extender la utilidad de los agentes antitrombóticos a la trombofilaxis. ${ }^{45,46}$

El diseño de moléculas de adhesión expresadas sobre la superficie en reposo y en las células endoteliales alteradas patológicamente permitirá la liberación de fármacos en la superficie endotelial (preferentemente para trombolíticos) y en compartimentos intracelulares (preferentemente para antioxidantes). Ya se emplean con cautela plataformas para la liberación dirigida de fármacos -incluyendo proteínas conjugadas, constructos de fusión recombinante y acarreadores poliméricos - a nivel del endotelio. ${ }^{47}$

\section{Endotelio vascular}

Las alteraciones endoteliales están implicadas en varias patologías, incluyendo enfermedades comunes agudas con alta morbimortalidad debido en parte a que los fármacos y los acarreadores de fármacos no tienen afinidad natural con el endotelio vascular. Ligandos de moléculas expuestos a la corriente

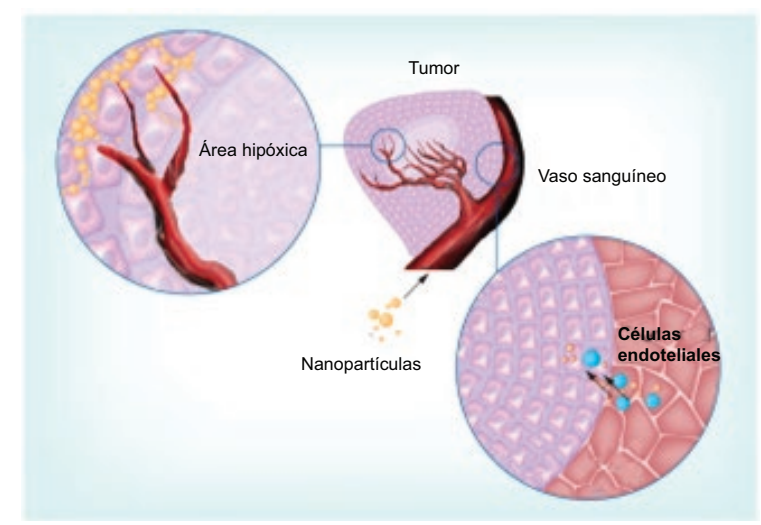

Figura 4. Aplicación de nanopartículas y sus efectos en células endoteliales.

sanguínea en la superficie endotelial permitirán diseñar agentes terapéuticos específicos dirigidos a nivel endotelial.

La molécula blanco y la unión a epítopes deben ser accesibles a los acarreadores de fármacos, los cuales deben estar libres de efectos dañinos; la elección del sitio blanco proporciona el direccionamiento deseable a nivel subcelular para la llegada del fármaco. La lista de las moléculas blanco candidatas para la aplicación de nanomedicina endotelial incluye peptidasas y otras enzimas, moléculas de adhesión celular e integrinas, localizadas en diferentes dominios de la membrana plasmática endotelial y diferencialmente distribuidas en todo el sistema vascular. Dotar a los acarreadores con una afinidad a los epítopes endoteliales específicos permitirá un nivel sin precedentes para la precisión y el control de la liberación de fármacos: unión a fenotipos de células endoteliales seleccionadas, la conducción celular y la duración de los efectos terapéuticos. Las características de los nanoacarreadores dependerán de la elección del epítope, el control de la liberación del ligando y el efecto de los agentes nanomédicos endoteliales específicos. La selección de los sitios de unión óptimos y las características de los nanoacarreadores son factores clave en estudios in vitro y modelos experimentales preclínicos in vivo. Los agentes nanomédicos dirigidos al endotelio proporcionan efectos antioxidantes, antiinflamatorios y otros efectos terapéuticos en modelos animales ${ }^{48}$ (Figura 4).

Por otra parte, la nanotecnología dirigida e individualizada promete encontrar usos clínicos en diversas áreas médicas. Varios marcadores de superficie endotelial, incluyendo peptidasas (por ejemplo, ACE, APP y APN) y moléculas de adhesión (por ejemplo,ICAM-1 y PECAM) han sido identificadas como objetivos claves. La unión de nanoacarreadores a estas moléculas debe ser capaz de permitir transportar 
fármacos y la posterior penetración del endotelio. El análisis de diversos aspectos de la nanomedicina endotelial deberá incluir:

- Circulación y orientación de los acarreadores con diversas geometrías.

- Interacciones multivalentes del acarreador con el endotelio.

- Anclaje a múltiples determinantes.

- Accesibilidad a los sitios de unión y respuesta celular a su compromiso.

- Papel del fenotipo celular y microambiente en la elección del sitio blanco.

- Optimización de la orientación por disminución de la avidez de los acarreadores.

- Endocitosis de acarreadores multivalentes vía moléculas no implicadas en la internalización de ligandos.

- Modulación de la captación y tráfico celular por selección de epítopes específicos en sitios determinantes, geometría del acarreador y factores hidrodinámicos. ${ }^{49}$

El refinamiento de estos aspectos mejorará el entendimiento de la fisiopatología vascular y deberá aplicarse a nivel clínico para la conducción de nanoacarreadores en el endotelio vascular.

La tecnología de reconocimiento multidimensional de proteínas es empleada para identificar varias proteínas (con tres o más espectros) ${ }^{50}$ en las membranas plasmáticas de las células endoteliales luminales aisladas de cultivo de células endoteliales microvasculares de animales.

Finalmente, el mapeo y caracterización de la vasculatura endotelial usando proteómica ofrece la oportunidad de una mayor comprensión de las etapas y mecanismos moleculares en el desarrollo vascular y la angiogénesis. La proteómica tiene ventajas importantes sobre la genómica, especialmente en la determinación de la expresión de proteínas. Es necesario centrarse en los principios y métodos proteómicos, con particular interés en sus aplicaciones para caracterizar al endotelio vascular (normal y tumoral), como el que existe in vivo. ${ }^{51}$

\section{Conclusiones}

Diversos métodos han sido diseñados para el ingreso inocuo de los nanoacarreadores en los vasos sanguíneos y el tejido cardiaco, de tal forma que los fármacos puedan ser dirigidos y liberados selectivamente, lo que incrementará su eficacia terapéutica y minimizará los efectos adversos. Se ha intensificado la utilización de nanoacarreadores y nanodispositivos en modelos animales in vivo e in vitro, lo que en un futuro cercano deberá traducirse en su aplicación clínica que disminuya significativamente la morbimortalidad de las ECV.

\section{Bibliografía}

1. Global status report on noncommunicable diseases 2010. Ginebra, Suiza: World Health Organization; 2011.

2. Mathers $C D$, Loncar D. Protections of global mortality and burden of disease from 2002 at 2030. PLoS Med. 2006;3(11):e442.

3. Tyler PD, Kang PM. Diagnostic and therapeutic nanoparticles in cardiovascular diseases. Curr Pharm Des. 2015;21(42):6070-6080.

4. Chauvierre C, Letourneur D. The European project NanoAthero to fight cardiovascular diseases using nanotechnologies. Nanomedicine (Lond). 2015; 10(22):3391-400.

5. Kransnoslobodtsev AV, Shlyakhtenko LS, Ukraintsev E, Zaikova TO, Keana JF, Lyubcchenko YL. Nanomedicine and protein misfolding diseases. Nanomedicine. 2005;1(4):300-305.

6. Binsalamah ZM, Paul A, Prakash S, Shum-Tim D. Nanomedicine in cardiovascular therapy: recent advancements. Expert Rev Cardiovasc Ther. 2012;10(6):805-815.

7. Bell IR, Ives JA, Jonas WB. Nonlinear effects of nanoparticles: biological variability from hermetic doses, small particle sizes, and dynamic adaptive interactions. Dose Response. 2013;12(2):202-232.

8. Wu J, Kamaly N, Shi J, Zhao L, Xiao Z, Hollett G, et al. Development of multinuclear polymeric nanoparticles as robust protein nanocarriers. Angew Chem Int Ed Engl. 2014;53(34):8975-8979.

9. Rodríguez-Vila B, Tarjuelo-Gutiérrez J, Sánchez-González $P$, Verbrugghe P, Fourneau I, Maleux G, et al. Automated delineation of vessel wall and thrombus boundaries of abdominal aortic aneurysms using multispectral MR images. Comput Math Methods Med. 2015;2015:202539.

10. Ferreira MP, Balasubramanian V, Hirvonen J, Ruskoaho H, Santos HA. Advanced nanomedicines for the treatment and diagnosis of myocardial infarction and heart failure. Curr Drug Targets. 2015;16(14):1682-1697.

11. Tang Y, Gan X, Cheheltani R, Curran E, Lamberti G, Krynska B, et al. Targeted delivery of vascular endothelial growth factor improves stem cell therapy in a rat myocardial infarction model. Nanomedicine. 2014;10(8):1711-1718.

12. Pu KM, Sava P, González AL. Microvascular targets for anti-fibrotic therapeutics. Yale J Biol Med. 2013;86(4):537-554.

13. Howard M, Zern BJ, Anselmo AC, Shuvaev VV, Mitragotri S, Muzykantov V. Vasculartargeting of nanocarriers: perplexing aspects of the seemingly straightforward paradigm. ACS NANO. 2014;8(5): 4100-4132.

14. Gadde S, Even-Or O, Kamaly N, Hasija A, Gagnon PG, Adusumilli KH, et al. Development of therapeutic polymeric nanoparticles for the resolution of inflammation. Adv Health Mater. 2014;3(9):1448-1456.

15. Marrache S, Pathnak RK, Darley KL, Choi JH, Zaver D, Kolishetti N, et al. Nanocarriers for tracking and treating diseases. Curr Med Chem. 2013;20(28):3500-3514

16. Howard MD, Hood ED, Zern B, Shuvaev VV, Grosser T, Muzykantov VR. Nanocarriers for vascular delivery of anti-inflammatory agents. Annu Rev Pharmacol Toxicol. 2014;54:205-226.

17. Shuvaev VV, Han J, Tliba S, Arguiri E, Christofidou-Solomidou M, Ramírez SH, et al. Anti-inflammatory effect of targeted delivery of SOD to endothelium: mechanism, synergism with NO donors and protective effects in vitro and in vivo. PLoS One. 2013;8(10):e77002.

18. Schiener M, Hossann M, Viola JR, Ortega-Gomez A, Weber C, Lauber K, et al. Nanomedicine-based strategies for treatment of atherosclerosis. Trends Mol Med. 2014;20(5):271-281.

19. Marrache S, Dhar S. Biodegradable synthetic high-density lipoprotein nanoparticles for atherosclerosis. Proc Natl Acad Sci USA. 2013;110(23):9445-9450.

20. Karagkiozaki V, Logothetidis S, Pappa AM. Nanomedicine for atherosclerosis: molecular imaging and treatment. J Biomed Nanotechnol. 2015;11(2):191-210.

21. Lewis DR, Petersen LK, York AW, Zablocki KR, Joseph LB, Kholodovych V, et al. Sugar-based amphiphilic nanoparticles arrest atherosclerosis in vivo. Proc Natl Acad Sci U S A. 2015;112(9):2693-2698.

22. Lanza GA. Angina pectoris and myocardial ischemia in the absence of obstructive coronary artery disease: role of diagnostic tests. Curr Cardiol Rep. 2016;18(2):15.

23. Mulder WJ, Jaffer FA, Fayad ZA, Nahrendorf M. Imaging and nanomedicine in inflammatory atherosclerosis. Sci Transl Med. 2014;6(239):239sr1.

24. Khalyfa A, Gozal D. Exosomal miRNAs as potential biomarkers of cardiovascular risk in children. J Transl Med. 2014;12:162. 
25. Du AW, Stenzel MH. Drug carriers for the delivery of therapeutic peptides. Biomacromolecules. 2014;15(4):1097-1114.

26. Yin RX, Yang DZ, Wu JZ. Nanoparticle drug- and gene-eluting stents for the prevention and treatment of coronary restenosis. Theranostics. 2014;4(2):175-200.

27. Karagkiozaki V, Karagiannidis PG, Kalfagiannis N, Kavatzikidou P, Patsalas $\mathrm{P}$, Georgiou D, et al. Novel nanostructured biomaterials: implications for coronary stent thrombosis. Int J Nanomedicine. 2012;7: 6063-6076.

28. Rolim T, Cancino J, Zucolotto V. A nanostructured genosensor for the early diagnosis of systemic arterial hypertension. Biomed Microdevices. 2015;17(1):3.

29. Lanza GM, Moonen C, Baker JR, Chang E, Cheng Z, Grodzinski P, et al Assessing the barriers to image-guided drug delivery. Wiley Interdiscip Rev Nanomed Nanobiotechnol. 2014:6(1):1-14.

30. Weissleder R, Nahrendorf M. Advancing biomedical imaging. Proc Natl Acad Sci U S A. 2015;112(47):14424-14428.

31. Godin B, Sakamoto JH, Serda RE, Grattoni A, Bouamrani A, Ferrari M Emerging applications of nanomedicine for the diagnosis and treatment of cardiovascular diseases. Trends Pharmacol Sci. 2010;31(5):199-205.

32. Aguirre $A D$, Vinegoni $C$, Sebas M, Weissleder R. Intravital imaging of cardiac function at the single-cell level. Proc Natl Acad Sci U S A. 2014;111(31):11257-11262.

33. Vinegoni $C$, Lee $S$, Aguirre AD, Weissleder R. New techniques for motion-artifact-free in vivo cardiac microscopy. Front Physiol. 2015;6:147.

34. Danila D, Johnson E, Kee P. CT imaging of myocardial scars with collagen-targeting gold nanoparticles. Nanomedicine. 2013;9(7):1067-1076.

35. Chrastina A, Massey KA, Schnitzer JE. Overcoming in vivo barriers to targeted nanodelivery. Wiley Interdiscip Rev Nanomed Nanobiotechnol. 2011;3(4):421-437.

36. Torchilin VP. Multifunctional, stimuli-sensitive nanoparticulate systems for drug delivery. Nat Rev Drug Discov. 2014;13(11):813-827.

37. Giljohann DA, Seferos DS, Daniel WL, Massich MD, Patel PC, Mirkin CA Gold nanoparticles for biology and medicine. Angew Chem Int Ed Engl. 2010;49(19):3280-3294.

38. Jordan C, Shuvaev VV, Bailey M, Muzykantov VR, Dziubla TD. The role of carrier geometry in overcoming biological barriers to drug delivery. Curr Pharm Des. 2016;22(9):1259-1273.
39. Sundararaj SC, Al-Sabbagh M, Rabek CL, Dziubla TD, Thomas MV, Puleo DA. Comparison of sequential drug release in vitro and in vivo. J Biomed Mater Res B Appl Biomater. 2016;104(7):1302-1310.

40. Ambati J, López AM, Cochran D, Wattamwar P, Bean K, Dziubla TD, et al. Engineered silica nanocarriers as a high-payload delivery vehicle for antioxidant enzymes. Acta Biomater. 2012;8(6):2096-2103.

41. Hood E, Simone E, Wattamwar P, Dziubla T, Muzykantov V. Nanocarriers for vascular delivery of antioxidants. Nanomedicine (Lond). 2011;6(7):1257-1272.

42. Kyle S, Saha S. Nanotechnology for the detection and therapy of stroke. Adv Healthc Mater. 2014;3(11):1703-1720.

43. Korin N, Kanapathipillai M, Matthews BD, Crescente M, Brill A, Mammoto T, et al. Shear-activated nanotherapeutics for drug targeting to obstructed blood vessels. Science. 2010;337(6095):738-742.

44. Psarros C, Lee R, Margaritis M, Antoniades C. Nanomedicine for the prevention, treatment and imaging of atherosclerosis. Maturitas. 2012;73(1):52-60.

45. Greineder CF, Howard MD, Carnemolla R, Cines DB, Muzykantov VR. Advanced drug delivery systems for antithrombotic agents. Blood. 2013;122(9):1565-1575.

46. Wadajkar AS, Santimano S, Rahimi M, Yuan B, Banerjee S, Nguyen KT. Deep vein thrombosis: current status and nanotechnology advances. Biotechnol Adv. 2013;31(5):504-513.

47. Carnemolla R, Shuvaev VV, Muzykantov VR. Targeting antioxidant and antithrombotic biotherapeutics to endothelium. Semin Thromb Hemost. 2010;36(3):332-342

48. Shuvaev VV, Brenner JS, Muzykantov VR. Targeted endothelial nanomedicine for common acute pathological conditions. J Control Release. 2015;219:576-595

49. Korin N, Gounis MJ, Wakhloo AK, Ingber DE. Targeted drug delivery to flow-obstructed vessels using mechanically activated nanotherapeutics. JAMA Neurol. 2015;72(1):119-122.

50. Durr E, Yu J, Krasinska KM, Carver LA, Yates JR, Testa JE, et al. Direct proteomic mapping of the lung microvascular endothelial cell surface in vivo and in cell culture. Nat Biotechnol. 2004;22(8):985-992.

51. Griffin NM, Schnitzer JE. Chapter 8. Proteomic mapping of the vascular endothelium in vivo for vascular targeting. Methods Enzymol. 2008;445: 177-208. 